\title{
Análise de taninos totais em plantas aromáticas e medicinais cultivadas no Nordeste do Rio Grande do Sul
}

\author{
Pansera, M.R ${ }^{1 *}$;; Santos, A.C.A. ${ }^{1,2}$; Paese, K. ${ }^{1}$; Wasum, R. ${ }^{1,4}$; Rossato, M. ${ }^{1,2 ;}$ Rota, \\ L.D. 1; Pauletti, G.F. ${ }^{1}$; Serafini, L.A. ${ }^{1,3}$ \\ ${ }^{1}$ Instituto de Biotecnologia, Universidade de Caxias do Sul; ${ }^{2}$ Departamento de Ciências Exatas e da \\ Natureza, Campus da Região de Vinhedos; ${ }^{3}$ Departamento de Física e Quimica, Centro de Ciências \\ Exatas e Tecnologia; ${ }^{4}$ Herbário da Universidade de Caxias do Sul, Caxias do Sul, RS.
}

RESUMO: Os taninos são componentes polifenólicos encontrados em plantas, alimentos e bebidas, sendo utilizados como fonte de matéria-prima para várias indústrias, como por exemplo a farmacêutica e a alimentícia, no curtimento do couro, na produção de borrachas, no tratamento de água em barragens, entre outras. Em vista da importância deste grupo de compostos, algumas plantas aromáticas e medicinais cultivadas no Núcleo Agronômico do Instituto de Biotecnologia da Universidade de Caxias dos Sul foram avaliadas quanto ao teor de taninos totais: Angelica archangelica, Cinnamomun camphora var. linaloolifera fugita, Cymbopogon citratus, Lavandula dentata, Lippia alba, Rosmarinus officinalis e Thymus vulgaris, e esses foram comparados com o teor de taninos totais da Acacia mearnsii (acácia negra), planta utilizada para a extração de taninos. O processo de extração empregado foi a maceração com metanol e a quantificação foi realizada por espectrofotometria, utilizando-se o reagente de Folin Denis. A partir da curva de calibração (\% equivalente ao ácido tânico) foram obtidos os teores de taninos totais, que variaram de 0,01\% para Cymbopogom citratus a 18,9\% para Lippia alba.

Unitermos: plantas medicinais; taninos; espectrofotometria; reagente de Folin Denis.

ABSTRACT: Determination of tannin total content in aromatic and medicinal plants cultivated in Northern Rio Grande do Sul State, Brazil. Tannins are polyphenolic compounds present in plants which are also found in foods and beverages. They are specially used by pharmaceutical, food, leather and rubber industries, and also for water treatment. Considering the significance of this group of compounds, several of aromatic and medicinal plants cultivated in Northern Rio Grande do Sul State, Brazil, were tested for their total tannin content. Angelica archangelica, Cinnamomun camphora var. linaloolifera fugita, Cymbopogon citratus, Lavandula dentata, Lippia alba, Rosmarinus officinalis and Thymus vulgaris were analysed and compared with Acacia mearnsii (black wattle) which is industrially used for tannin extraction. The extraction procedure was made by macerating the plants in methanol, and the quantification was done using Folin Denis reagent and spectrophotometry. By using a calibration curve (\% tannic acid equivalent) the total tannin contents range from $0.01 \%$ (Cymbopogom citratus) to $18.9 \%$ (Lippia alba).

Key words: medicinal plants; tannins; spectrofotometry; Folin Denis reagent. 


\section{INTRODUÇÃO}

Os taninos são componentes polifenólicos distribuídos em plantas, alimentos e bebidas (MAKKAR; BECKER, 1998; SANTOS et al. 1997). De acordo com Zucker (1983), os taninos encontram-se distribuídos em plantas superiores, ocorrendo aproximadamente em $30 \%$ das famílias. Eles são solúveis em água e em solventes orgânicos polares, sendo capazes de precipitar proteínas (HARTISH; KOLODZIEJ, 1997).

Tais compostos são responsáveis pela adstringência de muitos frutos e plantas em geral, através da complexação entre taninos e proteínas, que é a base de algumas de suas propriedades biológicas, tais como controle de insetos, fungos e bactérias (AERTS et al. 1999).

Plantas ricas em taninos são empregadas na medicina tradicional no tratamento de diversas moléstias, tais como diarréias, hipertensão arterial, reumatismo, hemorragias, feridas, queimaduras, problemas estomacais, renias e do sistema urinário, e processos inflamatórios em geral (SANTOS; MELLO, 1999). Para tratar feridas, queimaduras e inflamações, o poder anti-séptico dos taninos pode ser explicado por sua capacidade de precipitar as proteínas das células superficiais das mucosas e dos tecidos, formando uma camada protetora (complexo tanino-proteína e/ou polissacarídeo) sobre a pele ou mucosa danificada, impedindo, assim, o desenvolvimento de microrganismos (HASLAM, 1996).

Os taninos são usados na indústria alimentícia como antioxidantes nos sucos de frutas e bebidas e como clarificante de vinhos; como corantes têxteis; na produção de borrachas; e como coagulantes e floculantes no tratamento de água em barragens (SANTOS; MELLO, 1999). Também podem ser aplicados no curtimento do couro (VICKERY;VICKERY, 1981), que baseia-se na associação dos polifenóis com a proteína colágeno (KHANDABABAEE; REE, 2001).

Neste trabalho, foram quantificados os taninos totais presentes em algumas espécies de plantas aromáticas e medicinais cultivadas: Angelica archangelica, Cinnamomun camphora var. linaloolifera fugita, Cymbopogon citratus, Lavandula dentata, Lippia alba, Rosmarinus officinalis e Thymus vulgaris,, visando avaliar a possibilidade de utilização dessas espécies como fonte de taninos para a indústria. As espécies acima mencionadas apresentaram bom desenvolvimento a campo e já são utilizadas para extração de óleos essenciais; por outro lado, é importante o estudo completo de espécies vegetais, a fim de que se conheça suas reais potencialidades, no que diz respeito à possibilidade de obtenção de substâncias ativas com aplicações comerciais.

\section{MATERIAIS E MÉTODOS}

\section{Obtenção e preparo do material vegetal}

As plantas foram coletadas no campo experimental do Núcleo Agronômico do Instituto de Biotecnologia da Universidade de Caxias do Sul (Rio Grande do Sul, S 29¹0.122' WO51 ${ }^{\circ}$ 11.118'), no período de janeiro a maio de 2002. As espécies foram identificadas pelo botânico Ronaldo Wasum, e as exsicatas foram depositadas no Herbário da Universidade de Caxias do Sul (HUCS), com os números 21.229 (Lavandula dentata L.), 12.891, (Cinnamomun camphora var. linaloolifera fujita), 21.228 (Rosmarinus officinalis L.), 21.230 (Thymus vulgaris L.), 21.226 (Angelica archangelica L.), 21.227 (Cymbopogon citratus Stapf.), 13.705 (Lippia alba (Mill.) 
N.E.Br.) e 12.674 (Acacia mearnsii). O material utilizado para os ensaios foi seco a $36^{\circ} \mathrm{C}$, durante dez dias, em estufa de secagem.

\section{Obtenção dos extratos brutos}

Cerca de $5 \mathrm{~g}$ de cada planta, seca e moída, foram acondicionados em erlenmeyers de $500 \mathrm{~mL}$, adicionando-se $50 \mathrm{~mL}$ de metanol, e permanecendo em repouso durante dez dias, segundo metodologia descrita por Cechinel Filho e Yunes (1998). Após este tempo, os extratos brutos metanólicos (=extratos totais) foram evaporados sob pressão reduzida.

\section{Determinação do rendimento dos extratos brutos}

Para determinação da quantidade de extrato obtido (=extrato total), para cada planta, foi utilizada a equação: TEA $(\%)=\frac{M_{f}}{M_{i}} \times 100$, onde TEA=teor de extrato total (\%); $M i=$ massa inicial da amostra (g); Mf= massa final do extrato seco $(\mathrm{g})$.

\section{Determinação quantitativa dos teores de taninos totais nos extratos}

Os teores de taninos totais nos extratos foram obtidos por dissolução de cada extrato bruto metanólico $(250 \mathrm{mg})$ em água destilada $(500 \mathrm{~mL})$. Numa alíquota dessa mistura $(2 \mathrm{~mL})$, foi adicionado o reagente de Folin-Denis $(2 \mathrm{~mL})$ e a solução resultante foi agitada vigorosamente e deixada em repouso por $3 \mathrm{~min}$. Uma solução aquosa de carbonato de sódio a $8 \%(2 \mathrm{~mL})$ foi adicionada à mistura, agitada e deixada em repouso por $2 \mathrm{~h}$. Após este tempo, a amostra foi centrifugada a $2000 \mathrm{rpm}$ para a remoção de materiais em suspensão. Para quantificação dos taninos totais no extrato foram preparadas soluções de 0,$004 ; 0,01 ; 0,02 ; 0,04 ; 0,1$ e $0,2 \mathrm{~g} / \mathrm{mL}$ de ácido tânico diluído em água. A absorvância foi medida a $725 \mathrm{~nm}$, e um branco foi utilizado a cada leitura.A partir dos resultados obtidos foi construída a curva de calibração analítica, utilizada posteriormente para o cálculo dos teores de taninos totais nas plantas em estudo (SEIGLER et al, 1986). As leituras foram feitas em espectrofotômetro Beckman DU530, tendo sido realizadas três repetições por amostra.

\section{Determinação quantitativa dos teores de taninos totais nas plantas}

Para o cálculo da concentração de taninos totais, em cada planta, foi utilizada a equação:

TTP $(\%)=$ TEA $\times$ TTE $\div 100$, onde TTP = teor de taninos totais na planta $(\%)$; TEA = teor de extrato total $(\%)$; TTE $=$ teor de taninos totais no extrato $(\%)$.

\section{RESULTADOS E DISCUSSÃO}

\section{Determinação da porcentagem de taninos totais nos extratos}

Para a determinação do teor de taninos totais nos extratos, utilizou-se a equação da curva de calibração: $y=0,00144$.x, obtida a partir das diferentes concentrações de ácido tânico padrão. O padrão de ácido tânico foi utilizado por ser um tanino hidrolisável e, também, por caracterizar os taninos totais pelo método espectrofotométrico. A curva de calibração obtida encontra-se na Figura 1. 


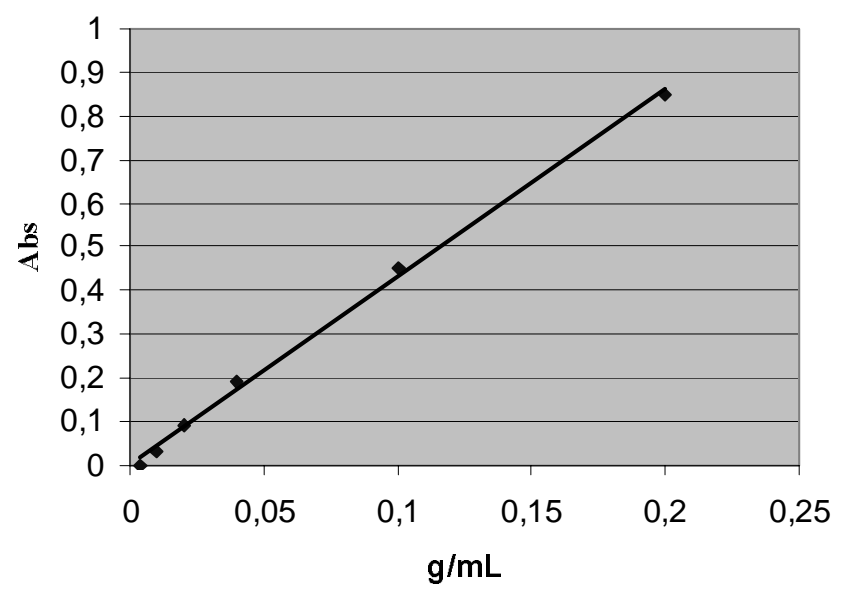

Figura 1. Curva de calibração de taninos totais - concentração de ácido tânico em $\mathrm{g} / \mathrm{mL}$ versus absorvância (Abs); Equação da reta: Absorvância = a. $c+b$; Erro do ajuste: 0,998888

Em relação ao método de extração, observou-se que o mesmo foi bastante efetivo para a extração dos taninos totais. Conforme Scalbert et al. (1989), para que a extração de taninos com metanol seja eficaz, deve-se aplicá-lo à baixas temperatura e pressão. Com este solvente de extração, a probabilidade de se extrair elagitaninos é maior do que a de extrair galotaninos, no caso, o ácido tânico.

A Tabela 1 mostra valores resultantes da quantificação dos taninos totais (\% equivalente ao ácido tânico) presentes nas plantas. Os teores variaram de 0,01\% (v/p) para Cymbopogom citratus a $18,9 \%$ (v/p) para Lippia alba.

Comparando-se os resultados obtidos com os dados de literatura (TRUGILHO et al. 2002), pode-se observar que Lippia alba apresentou um rendimento satisfatório, visto que espécies conhecidas pelos seus elevados teores de taninos, apresentam no máximo $20 \%$ de taninos totais, como é o caso da goiabeira (Psidium guajava: 13 a 17\%) e do araçá-pitanga (Psidium ruum: 20\%). A Acacia mearnsii (acácia-negra) é uma das espécies com maior potencial para utilização industrial da casca na produção de taninos, sendo empregada no curtimento do couro, apresentando $14 \%$ de taninos totais na casca (PANSERA et al. 2001). 0 fato da Lippia alba apresentar $18,9 \%$ de taninos justificaria a possível substituição da Acacia mearnsii, haja visto que a coleta das cascas impede o crescimento do vegetal, além do longo período que as árvores levam para crescer, podendo ser substituídas por arbustos, que apresentam crescimento mais rápido.

Ficou evidenciado, desta forma, que a quantidade de taninos totais é característica de cada espécie e não da quantidade total de extratos solúveis, pois as folhas da Lavandula dentata, apesar de terem apresentado uma das maiores solubilidades em metanol, produziram uma dos menores teores de taninos totais, não existindo, desta forma, uma correlação entre o teor de extratos totais e o teor de taninos totais na planta, mesmo porque, existem inúmeros outros compostos, que podem estar presentes no referido extrato. 
Tabela 1. Resultados quantitativos obtidos dos extratos das plantas aromáticas e medicinais cultivadas no Nordeste do Rio Grande do Sul.

\begin{tabular}{c|c|c||c|}
\hline Espécie & $\begin{array}{c}\text { Rendimento de } \\
\text { extrato total (\%) }\end{array}$ & $\begin{array}{c}\text { Taninos totais no } \\
\text { extrato } \\
\text { (\% equivalente ao } \\
\text { ácido tânico) }\end{array}$ & $\begin{array}{c}\text { Taninos totais na } \\
\text { planta } \\
\text { (\% equivalente ao } \\
\text { ácido tânico) }\end{array}$ \\
\hline Acacia mearnsii & 23,3 & 59,0 & 14,00 \\
\hline $\begin{array}{c}\text { Angelica archangelica } \\
\text { (folhas) }\end{array}$ & 20,1 & 11,0 & 2,20 \\
\hline Cinnamomun camphora & 18,3 & 10,0 & 1,80 \\
\hline Cymbopogom citratus & 14,7 & 15,0 & 0,01 \\
\hline Lavandula dentata & 30,0 & 14,0 & 4,20 \\
\hline Lippia alba & 45,0 & 42,0 & 18,90 \\
\hline Rosmarinus officinalis & 29,4 & 16,0 & 4,70 \\
\hline Thymus vulgaris & 12,9 & 26,0 & 3,30 \\
\hline \hline
\end{tabular}

\section{AGRADECIMENTOS}

Universidade de Caxias do Sul, Secretaria do Estado de Ciência e Tecnologia do Rio Grande do Sul, CAPES e CNPq

\section{REFERÊNCIAS BIBLIOGRÁFICAS}

AERTS, T.J.; BARRY, T.N.; MCNABB, W.C. Polyphenols and agriculture: beneficial effects of proanthocyanidins in forages. Agriculture, Ecosystems and Environment, v.75, p.1-12, 1999.

CECHINEL FILHO, V., YUNES, R.A. Estratégias para a obtenção de compostos farmacologicamente ativos a partir de plantas medicinais: conceitos sobre modificação estrutural para otimização da atividade. Química nova, v.21, p.99-105, 1998.

HARTISH, C.; KOLODZIEJ, H. Galloylhamameloses and proantocyanidins from Hamamelis virginiana. Phytochemistry, v.42, n.1, p.191-198, 1997.

HASLAM, E. Natural polyphenols (vegetable tannins) as drugs and medicines: possible modes of action. Journal of Natural Products, v.59, p.205-215, 1996.

KHANDABABAEE, K.; REE, T.V. Tannins: classification and definition. Nature Product Reports, v.18, p.641649, 2001.

MAKKAR, H.P.S.; BECKER, K. Do tannins in leaves of trees and shrubs from African and Himalayan regions differ in level and activity? Agroforestry Systems, v.40, p.59-68, 1998.

PANSERA, M. R.; ATTI-SANTOS, A.C.; SERAFINI, L.A.; CASSEL, E. Supercritical extraction of tannin with co-solvents. IV Encontro Brasileiro de Fluidos Supercríticos em Salvador-Bahia. 42, 2001.

SANTOS, S.A.; ABREU, L.R.; CHAGAS, S.J.R.. Efeito do armazenamento nos teores de fenólicos em caules de abacaxizeiro. Revista Brasileira de Agrociência, v.3, n. 3, p.107-109, 1997.

SANTOS, S. da C.; MELLO, J.C.P. de. Taninos In: SIMÕES, C.M.O.; SCHENKEL, E.P.; GOSMANN, G.; MELLO, J.C.P.; MENTZ, L.A.; PETROVICK, P.R. (Org.). Farmacognosia: da planta ao medicamento. Porto Alegre: Editora da Universidade; Florianópolis: EdUFSC, p.323-354, 1999. 
SCALBERT, A.; MONTIES, B.; JANNIN, G. Tannins in wood: comparison of different estimation methods. Journal of Agricultural and Food Chemistry, v.37, p.1324-1329, 1989.

SEIGLER, D.S.; SEILHEIMER, S.; KEESY, J.; HUANG, H.F. Tannins from four common Acacia species of Texas and Northeastern Mexico. Economic Botany, v.40, p.220-232, 1986.

TRUGILHO, P. F.; CAIXETA, R.P.; LIMA, J. T.; MENDES, L.M. (2002). Avaliação do conteúdo de taninos condensados de algumas espécies típicas do cerrado mineiro. Disponível em: <http: // www.dcf.ufla.br/ cerne/revistav3n1-1997/Publica\%c3\%87\%c3\%830.doc> Acesso em: 02 de junho de 2002.

VICKERY, M.L.; VICKERY, B. Secondary plant metabolism. London: Mc Millan, 1981.

ZUCKER, W.V. Tannins: does structure determine function? An ecological perspective. The American Naturalist, v.121, p.335-365, 1983.

*Autor para correspondência:

Profa. Dra. Marcia Regina Pansera

Instituto de Biotecnologia - Universidade de Caxias do Sul.

Rua Francisco Getúlio Vargas, 1130

95001-970 - Caxias do Sul - RS

E-mail: mrpanser@ucs.br 